\title{
A COMPUTER-CONTROLLED SYSTEM FOR ICE-FABRIC ANALYSIS ON A RIGSBY STAGE
}

by

Manfred A. Lange

(Alfred Wegener Institute for Polar and Marine Research,

Postfach 120161 , Columbusstraße, D - 2850 Bremerhaven, Federal Republic of Germany)

\begin{abstract}
An integrated system for ice-fabric analysis on a Rigsby stage is described. The system consists of a regular Rigsby stage fitted with two opto-electronic sensors for assessment of azimuth and the tilt angle of each individual grain. Signals from the sensors are transmitted to a computer terminal via an interface box, which facilitates transformation of Gray-coded data to ASCII data records. The terminal is hooked up to a main-frame computer (VAX 750), where the digitized angles of the $c$-axis orientations of individual thin sections are stored in separate data files. These files are compatible with other already existing files containing additional ice-core data and thus become part of an extensive data bank. Appropriate software has been developed to produce, among other things, plots of $c$-axis orientations in a Schmidt net.
\end{abstract}

\section{INTRODUCTION}

The orientation of the $c-$ (or the optical) axis of individual ice grains constitutes a fundamental parameter, providing insight into a number of basic ice properties. In particular, the distribution of $c$-axis orientations for a given thin section reveals information on the growth process and subsequent history of the sampled ice. In fresh-water ice, insight into the degree of maturation as well as the existence of large-scale deformations of a particular sample can be gained in this way. $c$-axis orientations are of ten related to other physical properties of an ice core and can be used as a predictive tool, provided a reliable correlation between $c$-axis orientation and the property under consideration has been defined.

In sea ice, determination of $c$-axis orientation mainly serves the purpose of identifying essential growth processes, as well as helping to assign particular ice classes to individual ice floes.

Assessing the internal structure of an ice sheet by means of crystal-fabric analysis requires a substantial number of $c$-axis determinations to be made on a single ice core. With an increase in scientific activity in the polar regions, and with advanced drilling techniques, the number of ice cores and thus the number of analyses to be performed has steadily grown. As a consequence the determination of $c$-axes and the conventional manual handling of ice-core data becomes a time-consuming and cumbersome process. In particular, data analysis by means of modern computer methods requires that the data be input into the computer, and this proves to be more and more impractical with the increasing number of analyses performed. Thus there clearly exists the need for an automatic or semi-automatic system for $c$-axis determinations on ice thin sections.

However, fully automatic systems not only require a substantial financial investment, but also specialized equipment (such as X-ray sources; Mori and others 1985) which is not readily available to most investigators.

\begin{tabular}{|l|l|l|l|}
\hline $\begin{array}{l}\text { Rigsby stage } \\
\text { with opto-elec- } \\
\text { tronic sensors }\end{array}$ & $\begin{array}{l}\text { Interfoce: opto } \\
\text { electronic sen- } \\
\text { sors / VAX } \\
\text { (Gray code } \\
\text { converter) }\end{array}$ \\
\hline
\end{tabular}


Attempts to use ultrasonic measurements for the determination of $c$-axis distributions, although promising, need further refinement before reliable results can be obtained (Langway and others 1988). Thus semi-automatic equipment, consisting of electronic components which link directly with a computer or a data logger, appear to be a sensible compromise. The major advantage of such a system lies in the fact that it frees the operator from writing down numbers while simultaneously inputting the data into a computer (or into a computer-compatible data-storage medium) for further analysis. Morgan and others (1984) described such a semi-automatic system, which uses a dedicated mini-computer for data storage and analysis. We have recently developed a comparable system, which, however, differs from the apparatus of Morgan and others mainly in using a main-frame computer for data acquisition and data handling. This enables usage of extensive software and hardware in the data processing, and linkage of fabric data with other physical and chemical ice-core data.

\section{BASIC COMPONENTS OF THE COMPUTER- CONTROLLED INTEGRATED ICE-FABRIC ANALYSIS SYSTEM}

Our fabric-analysis system consists of three major components:

(i) a conventional Rigsby stage fitted with two opto-electronic sensors for measurement of azimuth and a tilt angle,

(ii) an interface, which converts the Gray-coded signals (a sequential binary code, also called "reflective code") of the two sensors into ASCII data and puts them on to an RS 232-exit, and

(iii) a VAX 750/11 main-frame computer with appropriate software, which receives and stores the data and is also used in subsequent data analysis (Fig.1).

\section{The Rigsby stage}

The basic component of our system is a conventional Rigsby universal stage (for a general description see Langway 1958). The stage has three axes of rotation: one

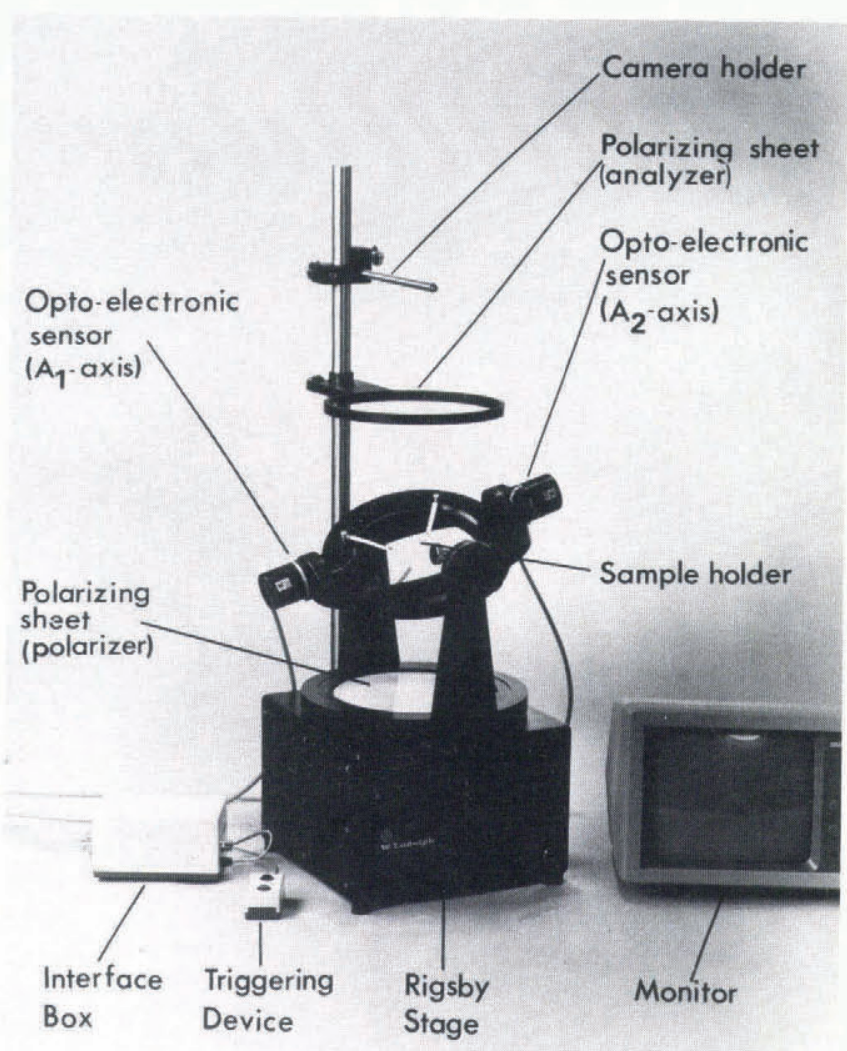

Fig.2. The major hardware components of the integrated ice-fabric analysis system. azimuthal rotation $\left(=A_{1}\right)$ and two axes of horizontal inclination, oriented in the north-south or east-west direction ( $=\mathrm{A}_{2}$ and $\mathrm{A}_{4}$ respectively). In addition, the entire stage can be rotated around a fourth, vertical, axis $\left(=A_{5}\right)$. In order to determine the orientation of the optic axis (or $c$-axis) of an individual grain within an ice thin section, rotation around these axes according to standard procedures (Langway 1958) will bring the grain to extinction, i.e. the grain then appears dark. Two cases have to be distinguished: either the optic axis coincides with the line of sight (polar position), or the optic axis is normal to the line of sight (equatorial position). In addition, of course, the azimuth angle $a_{1}$ and the inclination angle $a_{2}$ must be recorded (Langway 1958).

Figure 2 shows the Rigsby stage with its major components. Added to the standard stage are two optoelectronic devices that sense the rotation of the current setting around the $A_{1}$ and $A_{2}$ axes. The sensors employ GaAS-Diodes to scan a coded black-and-white pattern inside the sensor housing and produce a Gray-code digital signal of angles $a_{1}$ and $a_{2}$ between $0^{\circ}$ and $360^{\circ}$. The resolution of the sensors is $1^{\circ}$. The monitor seen in Figure 2 displays the current values of $a_{1}$ and $a_{2}$. A triggering device is used to initiate transmission of a setting of $a_{1}$ and $a_{2}$, via the interface box to the VAX. One of the buttons on the trigger is used for the equatorial and the other for the polar position of each axis.

The Rigsby stage, the monitor and the triggering device can all be operated in a cold-room, in temperatures as low as $-30^{\circ} \mathrm{C}$. To ensure proper operation of the television screen at these temperatures, it is housed in an insulated box which is fitted with a plexi-glass window for proper viewing of the screen.

\section{The interface box}

The major function of the interface box (placed outside the cold-room) is the conversion of the Gray-code signals into ASCII signals and their direct transmission (via an RS 232 interface) to the VAX computer. Figure 3 shows the major components of the interface and their functions. The Gray-code signals are steadily transformed into ASCII signals by the Gray-code converter and sent both to the VAX and to the monitor in order to control the movements of the Rigsby-stage axes $A_{1}$ and $A_{2}$. When one of the buttons on the triggering device is pushed, the micro-computer instructs the VAX to read the current readings into a data file. The transmitted data record includes a symbol A or B, which indicates either equatorial or polar positioning respectively, and two four-digit numbers, with symbols $\mathrm{X}$ and $\mathrm{Y}$ marking the $\mathrm{a}_{1}$ or $\mathrm{a}_{2}$ angle respectively of the $c$-axis being measured.

\section{Data handling and data analysis on the VAX}

Data handling and analysis require two or three steps. Data acquisition (including basic information on sample locality and sampling details) is facilitated by use of a program which organizes the sample- and angle data into separate files for each analyzed thin section. In a second step, which can be included in the first step, the angle data are transformed into a standard format where a positive $a_{1}$

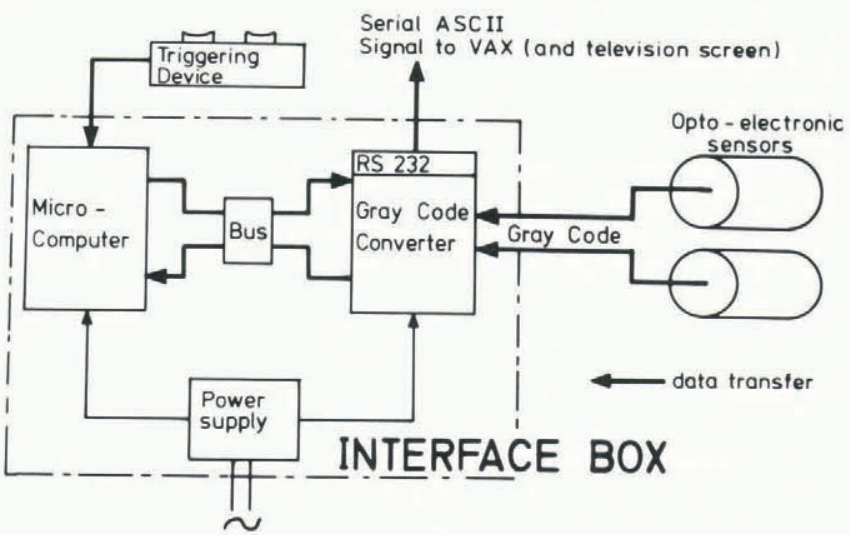

Fig.3. Basic elements of and relations between different components of the interface box (see text for details). 


\section{U1-Axis 期istributions:}

AN5331502

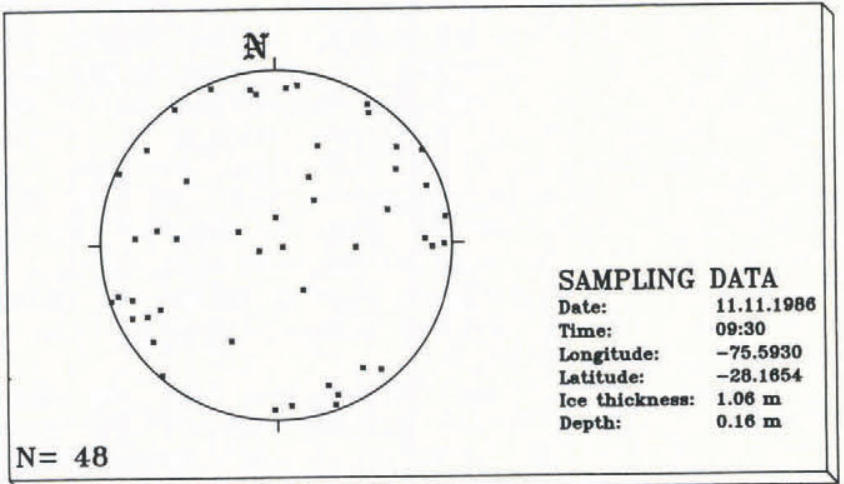

Fig.4. Sample Schmidt diagram of a sea-ice thin section (negative numbers for longitude and latitude represent west and south respectively).

means equatorial position, a negative $a_{1}$ means polar position, positive $a_{2}$ means tilt to the east and negative $a_{2}$ means tilt to the west. The $a_{2}$ values have to be corrected to eliminate the differences between observed and real angles, caused by the large differences between the refractive indices of air and ice. This is done by using formulations given by Kamb (1962).

The next step involves data handling. This includes a listing of $c$-axis orientations and their graphical representation in a Schmidt equal-area net (Fig.4). Subsequent programs yield fabric diagrams, i.e. contoured plots of the relative frequencies of $c$-axis orientations. It is also possible to produce plots of a $c$-axis distribution for different orientations, by computing the rotated angles of $c$-axes.

The files of the transformed and corrected $a_{1}$ and $a_{2}$ data are compatible with data files containing other physical and chemical ice-core data, such as densities, salinities and chlorophyll-a concentration, and can be merged with them. Thus we can create relatively extensive data banks of ice-core properties, including ice-fabric data, which can be easily handled and managed.

\section{PROCEDURES}

In order to determine the $c$-axis orientations on a thin section the following steps must be taken. First, the dataacquisition program has to be started on the VAX terminal, inputting the pertinent information on the sampled ice core and initiating the routine which inputs the orientation data. This is done outside the cold-room. The thin section to be analyzed is then placed on the Rigsby stage inside the cold-room. Orientations of single grains are determined manually by the operator, following standard procedures (Langway 1958). The final $a_{1}$ and $a_{2}$ values are sent to the VAX by pushing one of the buttons on the triggering device, depending on whether the grain is oriented in the polar or equatorial position. The polar or equatorial $c$-axis position has to be determined by the operator. The rotation angles can be read directly off the Rigsby stage, but they are also constantly displayed on the monitor. No noting of numbers is necessary, except for marking off on a thinsection photograph grains which have already been measured, thus speeding up the operation. A well-trained operator needs about $1 \mathrm{~min}$ to measure each grain orientation. When the desired number of grain orientations has been determined, the data-acquisition program has to be stopped on the terminal.

As the next step, the raw data have to be transformed and corrected before listings and plots can be obtained by running an additional program. Finally, the data can be combined with already existing data files for the same ice core. These steps usually only take a few minutes. Thus the total time required for a complete analysis (including thinsection preparation, $c$-axis determination and data handling) is usually about $1 \mathrm{~h}$.

\section{DISCUSSION}

The major advantages of this system are twofold. It greatly reduces the time the operator has to spend in the cold-room, as he is freed from taking notes and writing down numbers during the measurements. Although the absolute accuracy of each measurement, which is limited by the intrinsic mechanical accuracy of the Rigsby stage and by the observational accuracy (not better than $\pm 1-2^{\circ}$ for each angular reading), cannot be improved by the system, the risk of operator-induced errors is reduced.

The second advantage lies in the fact that the data are fed directly into a main-frame computer which has extensive peripheral devices. Although usage of the system is basically limited to laboratory investigations, it taps the extended capabilities of an integrated computer system. This also allows work to be done in an extensive, consistent data-bank system of ice-core data and enables automated comparisons of different ice properties in an individual ice core, as well as among a great number of comparable ones. Although this might sound trivial to an outside observer, investigators who have worked with large sets of ice-core data from different sources (or from different computer systems), will probably appreciate the advantages of an integrated system like the one described.

\section{ACKNOWLEDGEMENTS}

I should like to thank V Morgan (Australian Antarctic Division, Kingston, Tasmania) for providing valuable information on his own apparatus and for giving us useful hints on designing our system. I want to acknowledge the competent and dedicated work of $\mathrm{Mr}$ Frers and $\mathrm{Mr}$ Bünte of Firma Ludolph, Bremerhaven, in building the hardware components. I appreciate the help of $\mathrm{J}$ Schlüter of the Alfred Wegener Institute for Polar and Marine Research, Bremerhaven, who wrote the main part of the dataacquisition program. I thank two anonymous reviewers for very helpful suggestions. This is Alfred Wegener Institute contribution no. 46.

\section{REFERENCES}

Kamb W B 1962 Refraction correction for universal stage measurements. I. Uniaxial crystals. American Mineralogist 47: $227-245$

Langway C C Jr 1958 Ice fabrics and the universal stage. U.S. Army Snow, Ice and Permafrost Research Establishment. Technical Report 62

Langway C C Jr, Shoji H, Azuma N, 1988 Crystal size and orientation patterns in the Wisconsin-age ice from Dye 3, Greenland. Annals of Glaciology 10: 109-115

Morgan V I, Davis E R, Wehrle E 1984 A Rigsby Stage with remote computer compatible output. Cold Regions Science and Technology 10(1): 89-92

Mori Y, Hondoh T, Higashi A 1985 Development of an automatic ice fabric analyser. Annals of Glaciology 6: 281-283 